\title{
Novel Dark, Singular and Combo Optical Solitons for Fokas-Lenells Equation
}

\author{
M. LAKESTANi AND J. MANAFIAN* \\ Department of Applied Mathematics, Faculty of Mathematical Science, University of Tabriz, Tabriz, Iran \\ (Received February 22, 2019; in final form April 29, 2019)

\begin{abstract}
This paper retrieves new combo, dark and singular optical soliton solutions along with singular periodic, com-
\end{abstract} \\ bined hyperbolic and rational solutions to the Fokas-Lenells equation in birefringent fibres by integration tools such \\ as the improved $\tan (\phi(\xi) / 2)$-expansion method, improved Bernoulli sub-ODE method, and generalized $\left(G^{\prime} / G\right)$ - \\ expansion method. The existence criterions of these solutions are also listed.
}

DOI: 10.12693/APhysPolA.136.214

PACS/topics: Fokas-Lenells equation, improved $\tan (\phi(\xi) / 2)$-expansion method, improved Bernoulli sub-ODE method, generalized $\left(G^{\prime} / G\right)$-expansion method, soliton solutions

\section{Introduction}

In nonlinear optics, the advent of optical waveguides including optical fibres and crystals has engaged the researchers all over the world. Therefore, this work is carried out to study the dynamics of electromagnetic waves in birefringent fibres in the presence of differential group delay, an unavoidable feature due to the production irregularities and other glitches in fibres. The equation describing this behavior roots from the Fokas-Lenells equation (FLE) that can be capitalized to study polarizationpreserving fiber. The reputation of FLE has gained considerable hike since it is proposition in the previous decade. The soliton solutions of such type of models have comprehensively addressed in [1-28]. Some authors already have studied FLE in $[2-4,8,12,18,19]$. In the current research, the improved $\tan (\phi(\xi) / 2)$-expansion method and Bernoulli method are used to retrieve soliton solutions, rational function-like solutions, hyperbolic solutions, and periodic solutions for the FLE. This work is organized as, in next section a brief introduction of the proposed method is discussed. At the end some concluding remarks about the obtained results are given.

\section{Analysis model}

We consider the Fokas-Lenells equation (FLE) in presence of perturbation terms as

$$
\begin{aligned}
& \mathrm{i} \psi_{t}+\beta_{1} \psi_{x x}+\beta_{2} \psi_{x t}+|\psi|^{2}\left(b \psi+\mathrm{i} \sigma \psi_{x}\right)= \\
& \mathrm{i}\left(\alpha \psi_{x}+\lambda\left(|\psi|^{2} \psi\right)_{x}+\mu\left(|\psi|^{2}\right)_{x} \psi\right),
\end{aligned}
$$

where $\psi(x, t)$ represents a complex field envelope, and $x$ and $t$ are spatial and temporal variables, respectively [1]. The first term represents the linear evolution of the pulses in nonlinear optical fibres, while the coefficient

\footnotetext{
* corresponding author; e-mail j_manafianheris@tabrizu.ac.ir
}

$\beta_{1}$ is the spatiotemporal dispersion (STD) and $\beta_{2}$ is the group velocity dispersion (GVD). Then the fourth term introduces the cubic nonlinear term, while the fifth term accounts for dispersion. On the right hand side of Eq. (1), the coefficient of $\alpha$ is the inter-modal dispersion (IMD), while $\lambda$ is the selfsteepening perturbation term, and finally $\mu$ is the nonlinear dispersion (ND) coefficient.

To solve Eq. (1), the following hypothesis is considered:

$$
\psi(x, t)=u(\xi) \mathrm{e}^{\mathrm{i} \eta(x, t)}, \quad \xi=x-\nu t,
$$

and

$$
\eta=-k x+\omega t+\theta,
$$

Here, $\nu$ is the velocity of the soliton, $k$ is the frequency while $\omega$ is the soliton wave number, and $\theta$ is the phase constant to be determined. Employing Eq. (2) into Eq. (1) we get the following pair of equations of real and imaginary components, respectively as:

$$
\begin{aligned}
& \left(\beta_{1}-\beta_{2} \nu\right) u^{\prime \prime}-\left(\alpha k+\beta_{1} k^{2}-\beta_{2} k \omega\right) u \\
& \quad+(b-k \lambda+k \sigma) u^{3}=0
\end{aligned}
$$

and

$\left(\nu+\alpha+2 \beta_{1} k-\beta_{2}(\nu k+\omega)+(3 \lambda+2 \mu-\sigma) u^{2}\right) u^{\prime}=0$.

From (4), the velocity of the soliton is acquired as

$$
\nu=\frac{\alpha+2 \beta_{1} k-\beta_{2} \omega}{\beta_{2} k-1},
$$

such that $\beta_{2} k \neq 1$. The constraint condition has been obtained as

$$
3 \lambda+2 \mu-\sigma=0 \text {. }
$$

\section{Description of ITEM}

In this section, we describe the ITEM [27] with the following steps as:

Step 1. Let us consider a general form of the nonlinear partial differential equation 


$$
\mathcal{F}\left(u, u_{x}, u_{t}, u_{x x}, u_{t t}, \ldots\right)=0,
$$

where $F$ is a function of $u(x, t), u_{t}(x, t), u_{x}(x, t)$, $u_{x x}(x, t), \ldots$ including nonlinear terms, and the subscripts denote the partial derivatives.

The travelling wave variable transformation is set, that is, $u(x, t)=u(\xi), \xi=x-\nu t$, where $\nu$ is arbitrary constant. Then Eq. (7) is converted into an ODE as follows

$$
\mathcal{Q}\left(u, u^{\prime},-\nu u^{\prime}, u^{\prime \prime}, \nu^{2} u^{\prime \prime}, \ldots\right)=0,
$$

where $Q$ is a function of $u, \frac{\mathrm{d} u}{\mathrm{~d} \xi}, \frac{\mathrm{d}^{2} u}{\mathrm{~d} \xi^{2}}, \ldots$ with nonlinear terms.

Step 2. Let us consider the solution of Eq. (26) as

$u(\xi)=\sum_{k=0}^{M} e_{k} \tan ^{k}\left(\frac{\Phi(\xi)}{2}\right)+\sum_{k=1}^{M} f_{k} \cot ^{k}\left(\frac{\Phi(\xi)}{2}\right)$,

where $e_{k}(0 \leq k \leq M), f_{k}(1 \leq k \leq M)$ are constants to be determined, such that $e_{M} \neq 0, f_{M} \neq 0$, and $\phi=\phi(\xi)$ satisfies the following NLODE:

$$
\phi^{\prime}(\eta)=a \sin (\phi(\eta))+b \cos (\phi(\eta))+c .
$$

Step 3. Determining $M$ usually can be accomplished by balancing the linear term(s) of highest order with the highest-order nonlinear term(s) in Eq. (8). Moreover, precisely, we define the degree of $u(\eta)$ as $D(u(\eta))=M$, which gives rise to degree of another expression as follows:

$$
\begin{aligned}
& D\left(\frac{\mathrm{d}^{q} u}{\mathrm{~d} \eta^{q}}\right)=M+q, \\
& D\left(u^{p}\left(\frac{\mathrm{d}^{q} u}{\mathrm{~d} \eta^{q}}\right)^{s}\right)=M p+s(M+q) .
\end{aligned}
$$

Step 4. Inserting (9) into Eq. (8) with the value of $M$ obtained in Step 3, collecting the coefficients of $\tan (\phi / 2)^{k}, \cot (\phi / 2)^{k}(k=0,1,2, \ldots, M)$, and then setting each coefficient to zero, we can get a set of overdetermined equations for $e_{0}, e_{k}, f_{k}(k=1,2, \ldots, M) a, b$, and $c$ with the aid of symbolic computation using Maple. We solve the algebraic equations, and then substitute $e_{0}$, $e_{1}, \ldots, e_{M}, f_{1}, \ldots, f_{M}, k, \omega$ in $(9)$.

Consider the following special solutions of Eq. (10):

Family 1: When $\Delta=a^{2}+b^{2}-c^{2}<0$ and $b-c \neq 0$, then $\phi(\xi)=2 \arctan \left(\frac{a}{b-c}-\frac{\sqrt{-\Delta}}{b-c} \tan \left(\frac{\sqrt{-\Delta}}{2} \tilde{\xi}\right)\right)$.

Family 2: When $\Delta=a^{2}+b^{2}-c^{2}>0$ and $b-c \neq 0$, then $\phi(\xi)=2 \arctan \left(\frac{a}{b-c}+\frac{\sqrt{\Delta}}{b-c} \tanh \left(\frac{\sqrt{\Delta}}{2} \tilde{\xi}\right)\right)$.

Family 3: When $a^{2}+b^{2}-c^{2}>0, b \neq 0$ and $c=0$, then $\phi(\xi)=2 \arctan \left(\frac{a}{b}+\frac{\sqrt{b^{2}+a^{2}}}{b} \tanh \left(\frac{\sqrt{b^{2}+a^{2}}}{2} \widetilde{\xi}\right)\right)$.

Family 4: When $a^{2}+b^{2}-c^{2}<0, c \neq 0$ and $b=0$, then $\phi(\xi)=2 \arctan \left(-\frac{a}{c}+\frac{\sqrt{c^{2}-a^{2}}}{c} \tan \left(\frac{\sqrt{c^{2}-a^{2}}}{2} \widetilde{\xi}\right)\right)$.

Family 5: When $a^{2}+b^{2}-c^{2}>0, b-c \neq 0$ and $a=0$, then $\phi(\xi)=2 \arctan \left(\sqrt{\frac{b+c}{b-c}} \tanh \left(\frac{\sqrt{b^{2}-c^{2}}}{2} \widetilde{\xi}\right)\right)$.

Family 6: When $a=0$ and $c=0$, then $\phi(\xi)=$ $\arctan \left(\frac{\mathrm{e}^{2 b \widetilde{\xi}}-1}{\mathrm{e}^{2 b \widetilde{\xi}}+1}, \frac{2 \mathrm{e}^{b \widetilde{\xi}}}{\mathrm{e}^{2 b \widetilde{\xi}}+1}\right)$.

Family 7: When $b=0$ and $c=0$, then $\phi(\xi)=$ $\arctan \left(\frac{2 \mathrm{e}^{a \widetilde{\xi}}}{\mathrm{e}^{2 a \widetilde{\xi}}+1}, \frac{\mathrm{e}^{2 a \widetilde{\xi}}-1}{\mathrm{e}^{2 a \widetilde{\xi}}+1}\right)$.

Family 8: When $a^{2}+b^{2}=c^{2}$, then $\phi(\xi)=$ $-2 \arctan \left(\frac{(b+c)(a \widetilde{\xi}+2)}{a^{2} \widetilde{\xi}}\right)$.

Family 9: When $c=a$, then $\phi(\xi)=$ $-2 \arctan \left(\frac{(a+b) \mathrm{e}^{b \widetilde{\xi}}-1}{(a-b) \mathrm{e}^{b \widetilde{\xi}}-1}\right)$.

Family 10: When $c=-a$, then $\phi(\xi)=$ $2 \arctan \left(\frac{\mathrm{e}^{b \widetilde{\xi}}+b-a}{\mathrm{e}^{b \widetilde{\xi}}-b-a}\right)$.

Family 11: When $b=-c$, then $\phi(\xi)=$ $-2 \arctan \left(\frac{a \mathrm{e}^{a \widetilde{\xi}}}{c \mathrm{e}^{a \widetilde{\xi}}-1}\right)$.

Family 12: When $b=0$ and $a=c$, then $\phi(\xi)=$ $-2 \arctan \left(\frac{c \widetilde{\xi}+2}{c \widetilde{\xi}}\right)$.

Family 13: When $a=0$ and $b=c$, then $\phi(\xi)=$ $2 \arctan (c \widetilde{\xi})$.

Family 14: When $a=0$ and $b=-c$, then $\phi(\xi)=$ $-2 \arctan \left(\frac{1}{c \widetilde{\xi}}\right)$,

Family 15: When $a=0$ and $b=0$, then $\phi(\xi)=c \widetilde{\xi}+C$.

Family 16: When $b=c$, then $\phi(\xi)=$ $2 \arctan \left(\frac{\mathrm{e}^{a \widetilde{\xi}}-c}{a}\right)$,

where $\widetilde{\xi}=\xi+C$.

\section{Algorithm of the ITEM}

By solving Eq. (1) by $\tan (\phi(\xi) / 2)$-expansion method, and by balancing the terms $u^{\prime \prime}$ and $u^{3}$ we obtain $M=1$. Then the following hypothesis is considered:

$$
u(\xi)=e_{0}+e_{1} \tan (\phi(\xi) / 2)+f_{1} \cot (\phi(\xi) / 2) .
$$

Putting (12) into Eq. (3) and comparing the terms, and then solving a system of nonlinear algebraic equations the following new results can be yielded as:

\section{Set 1:}

$$
\begin{aligned}
& b=-c, \quad k=-\frac{c}{\lambda-\sigma}, \quad e_{1}=0, \\
& \omega=\frac{\alpha\left(a^{2} \beta_{2}-1\right)(\lambda-\sigma)^{3}-c \beta_{2}\left(2 a^{2} \beta_{1}+\alpha\right)(\lambda-\sigma)^{2}-c^{2} \beta_{1}(\lambda-\sigma)-c^{3} \beta_{1} \beta_{2}}{(\lambda-\sigma)\left[\left(a^{2} \beta_{2}^{2}+1\right)(\lambda-\sigma)^{2}+2 c \beta_{2}(\lambda-\sigma)+\beta_{2}^{2} c^{2}\right]}, \\
& f_{1}=\frac{\left[a^{2} \beta_{2}\left(\alpha \beta_{2} e_{0}+\alpha e_{0}-\beta_{1} \beta_{2}\right)-\beta_{1}\right](\lambda-\sigma)^{2}+\left[a^{2} c \beta_{2} e_{0}\left(\alpha \beta_{2}-2 \beta_{1}\right)-2 c \beta_{1} \beta_{2}\right](\lambda-\sigma)-\beta_{1} \beta_{2}^{2} c^{2}\left(a^{2} e_{0}+1\right)}{\beta_{2} c a\left[\alpha\left(\beta_{2}+1\right)(\lambda-\sigma)^{2}+c\left(\alpha \beta_{2}-2 \beta_{1}\right)(\lambda-\sigma)-\beta_{1} \beta_{2} c^{2}\right]} .
\end{aligned}
$$


By using family 2, the hyperbolic exact solution will be as

$$
\begin{aligned}
& u_{1}(\xi)=e_{0}-\frac{2 f_{1} c}{a\left[1+\tanh \left(\frac{a}{2}\left(x+\frac{\alpha-\frac{2 \beta_{1} c}{\lambda-\sigma}-\beta_{2} \omega}{\frac{\beta_{2} c}{\lambda-\sigma}+1} t\right)\right)\right]}, \quad \text { (14) } \\
& \psi_{1}(x, t)=\left\{e_{0}-\frac{2 f_{1} c}{a\left[1+\tanh \left(\frac{a}{2}\left(x+\frac{\alpha-\frac{2 \beta_{1} c}{\lambda-\sigma}-\beta_{2} \omega}{\frac{\beta_{2} c}{\lambda-\sigma}+1} t\right)\right)\right]}\right\} \\
& \times \exp \left(\mathrm{i}\left(\frac{c}{\lambda-\sigma} x+\omega t+\theta\right)\right) .
\end{aligned}
$$

By using family 11, the rational kink exact solution will be as

$$
u_{2}(\xi)=e_{0}+f_{1}\left[\frac{1-c \exp \left(a\left(x+\frac{\alpha-\frac{2 \beta_{1} c}{\lambda-\sigma}-\beta_{2} \omega}{\frac{\beta_{2} c}{\lambda-\sigma}+1} t\right)\right)}{a \exp \left(a\left(x+\frac{\alpha-\frac{2 \beta_{1} c}{\lambda-\sigma}-\beta_{2} \omega}{\frac{\beta_{2} c}{\lambda-\sigma}+1} t\right)\right)}\right]
$$

$\psi_{2}(x, t)=$

$$
\begin{aligned}
& \left\{e_{0}+f_{1}\left[\frac{1-c \exp \left(a\left(x+\frac{\alpha-\frac{2 \beta_{1} c}{\lambda-\sigma}-\beta_{2} \omega}{\frac{\beta_{2} c}{\lambda-\sigma}+1} t\right)\right)}{a \exp \left(a\left(x+\frac{\alpha-\frac{2 \beta_{1} c}{\lambda-\sigma}-\beta_{2} \omega}{\frac{\beta_{2} c}{\lambda-\sigma}+1} t\right)\right)}\right]\right\} \\
& \times \exp \left(\mathrm{i}\left(\frac{c}{\lambda-\sigma} x+\omega t+\theta\right)\right) .
\end{aligned}
$$

\section{Set 2:}

$$
\begin{aligned}
& b=c, \quad k=\frac{c}{\lambda-\sigma}, \quad f_{1}=0, \\
& \omega=\frac{\alpha\left(a^{2} \beta_{2}-1\right)(\lambda-\sigma)^{3}+c \beta_{2}\left(2 a^{2} \beta_{1}+\alpha\right)(\lambda-\sigma)^{2}-c^{2} \beta_{1}(\lambda-\sigma)+c^{3} \beta_{1} \beta_{2}}{(\lambda-\sigma)\left[\left(a^{2} \beta_{2}^{2}+1\right)(\lambda-\sigma)^{2}-2 c \beta_{2}(\lambda-\sigma)+\beta_{2}^{2} c^{2}\right]}, \\
& e_{1}=\frac{\left[\beta_{1}-a^{2} \beta_{2}\left(\alpha \beta_{2} e_{0}+\alpha e_{0}-\beta_{1} \beta_{2}\right)\right](\lambda-\sigma)^{2}+\left[a^{2} c \beta_{2} e_{0}\left(\alpha \beta_{2}-2 \beta_{1}\right)-2 c \beta_{1} \beta_{2}\right](\lambda-\sigma)+\beta_{1} \beta_{2}^{2} c^{2}\left(a^{2} e_{0}+1\right)}{\beta_{2} c a\left[-\alpha\left(\beta_{2}+1\right)(\lambda-\sigma)^{2}+c\left(\alpha \beta_{2}-2 \beta_{1}\right)(\lambda-\sigma)+\beta_{1} \beta_{2} c^{2}\right]} .
\end{aligned}
$$

By using family 16 , the rational exponential exact solution will be as

$$
\begin{aligned}
& u_{3}(\xi)=e_{0}+e_{1} \frac{\exp \left(a\left(x+\frac{\alpha+\frac{2 \beta_{1} c}{\lambda-\sigma}-\beta_{2} \omega}{\frac{\beta_{2} c}{\lambda-\sigma}+1} t\right)\right)-c}{a}, \\
& \psi_{3}(x, t)=\left\{e_{0}+e_{1} \frac{\exp \left(a\left(x+\frac{\alpha+\frac{2 \beta_{1} c}{\lambda-\sigma}-\beta_{2} \omega}{\frac{\beta_{2} c}{\lambda-\sigma}+1} t\right)\right)-c}{a}\right\} \exp \left(\mathrm{i}\left(-\frac{c}{\lambda-\sigma} x+\omega t+\theta\right)\right) .
\end{aligned}
$$

\section{Set 3:}

$$
\begin{aligned}
a= & \pm \frac{e_{0}(b+c) P}{\sqrt{P \beta_{1} \beta_{2} \Xi}}, \\
\Xi & =k \beta_{1} \beta_{2}(b+c)\left(\left(b^{2}-c^{2}\right) e_{0}-1\right)(\lambda-\sigma)+(b+c)\left[e_{0}\left(b^{2}-c^{2}\right)\left(\alpha \lambda \beta_{2}-\alpha \sigma \beta_{2}+b \beta_{1} \beta_{2}-2 \lambda \beta_{1}+2 \sigma \beta_{1}\right)\right. \\
& \left.-\beta_{2} e_{0}^{3}(\lambda-\sigma)^{2}\left(b^{2}-c^{2}\right)-\beta_{1}\left(b \beta_{2}-2 \lambda+2 \sigma\right)\right] \\
k & =\frac{1}{\beta_{1} \beta_{2}\left(b^{2} e_{0}-c^{2} e_{0}-1\right)}\left(-2 \beta_{1}+\beta_{2} e_{0}^{3}\left(b^{2}-c^{2}\right)(\lambda-\sigma)-e_{0}\left(b^{2}-c^{2}\right)\left(\alpha \beta_{2}-2 \beta_{1}\right)+\sqrt{\Omega}\right), \\
\Omega & =\beta_{2}^{2} e_{0}^{6}(\lambda-\sigma)^{2}\left(b^{2}-c^{2}\right)^{2}-2 \beta_{2} e_{0}^{4}\left(b^{2}-c^{2}\right)^{2}\left(\alpha \lambda \beta_{2}-\alpha \sigma \beta_{2}+2 b \beta_{1} \beta_{2}-2 \lambda \beta_{1}+2 \sigma \beta_{1}\right) \\
& +4 \beta_{1} \beta_{2} e_{0}^{3}\left(b^{2}-c^{2}\right)\left(b \beta_{2}-\lambda+\sigma\right)+e_{0}^{2}\left(b^{2}-c^{2}\right)^{2}\left(\alpha^{2} \beta_{2}^{2}+4 \alpha \beta_{1} \beta_{2}^{2}+4 \beta_{1}^{2}\right) \\
& -4 \beta_{1} e_{0}\left(b^{2}-c^{2}\right)\left(-b^{2} \beta_{1} \beta_{2}^{2}+c^{2} \beta_{1} \beta_{2}^{2}+\alpha \beta_{2}^{2}+\beta_{1}\right), \\
& \beta_{2} e_{0}\left(b^{2}-c^{2}\right)\left(2 k \beta_{1}+\alpha\right)-k \beta_{1} \beta_{2}+\beta_{1} \\
\omega & \left(b^{2}-c^{2}\right) \beta_{2}^{2} e_{0} \\
P & =2 \beta_{2} e_{0}^{2}(b+c)(b-c)^{2}\left[\left(\alpha \beta_{2}(-\lambda+b+\sigma)-2 b \beta_{1}\right)(\lambda-\sigma)-\alpha(\lambda-\sigma)^{2}+b^{2} \beta_{1} \beta_{2}\right] \\
& -2 \beta_{1} e_{0}(b-c)\left[b^{2}+\left(1+b^{2}-c^{2}\right)(\lambda-\sigma)^{2}-2 b \beta_{2}(\lambda-\sigma)\right] . \quad f_{1}=\frac{1}{P} \sqrt{P \beta_{1} \beta_{2} \Xi},
\end{aligned}
$$


By utilizing family 1 , the periodic exact solution is

$$
\begin{aligned}
& u_{4}(\xi)=e_{0}+e_{1}\left(\frac{a}{b-c}-\frac{\sqrt{-\Delta}}{b-c} \tan \left(\frac{\sqrt{-\Delta}}{2}\left(x-\frac{\alpha+2 \beta_{1} k-\beta_{2} \omega}{\beta_{2} k-1} t\right)\right)\right] \\
& +f_{1}\left[\frac{a}{b-c}-\frac{\sqrt{-\Delta}}{b-c} \tan \left(\frac{\sqrt{-\Delta}}{2}\left(x-\frac{\alpha+2 \beta_{1} k-\beta_{2} \omega}{\beta_{2} k-1} t\right)\right)\right]^{-1}, \\
& \psi_{4}(x, t)=\left\{e_{0}+e_{1}\left[\frac{a}{b-c}-\frac{\sqrt{-\Delta}}{b-c} \tan \left(\frac{\sqrt{-\Delta}}{2}\left(x-\frac{\alpha+2 \beta_{1} k-\beta_{2} \omega}{\beta_{2} k-1} t\right)\right)\right]\right. \\
& \left.+f_{1}\left[\frac{a}{b-c}-\frac{\sqrt{-\Delta}}{b-c} \tan \left(\frac{\sqrt{-\Delta}}{2}\left(x-\frac{\alpha+2 \beta_{1} k-\beta_{2} \omega}{\beta_{2} k-1} t\right)\right)\right]^{-1}\right\} \mathrm{e}^{\mathrm{i}(-k x+\omega t+\theta)} .
\end{aligned}
$$

By using family 2, the dark soliton exact solution is

$$
\begin{aligned}
& u_{5}(\xi)=e_{0}+e_{1}\left[\frac{a}{b-c}+\frac{\sqrt{\Delta}}{b-c} \tanh \left(\frac{\sqrt{\Delta}}{2}\left(x-\frac{\alpha+2 \beta_{1} k-\beta_{2} \omega}{\beta_{2} k-1} t\right)\right)\right] \\
& +f_{1}\left[\frac{a}{b-c}+\frac{\sqrt{\Delta}}{b-c} \tanh \left(\frac{\sqrt{\Delta}}{2}\left(x-\frac{\alpha+2 \beta_{1} k-\beta_{2} \omega}{\beta_{2} k-1} t\right)\right)\right]^{-1}, \\
& \psi_{5}(x, t)=\left\{e_{0}+e_{1}\left[\frac{a}{b-c}+\frac{\sqrt{\Delta}}{b-c} \tanh \left(\frac{\sqrt{\Delta}}{2}\left(x-\frac{\alpha+2 \beta_{1} k-\beta_{2} \omega}{\beta_{2} k-1} t\right)\right)\right]\right. \\
& \left.+f_{1}\left[\frac{a}{b-c}+\frac{\sqrt{\Delta}}{b-c} \tanh \left(\frac{\sqrt{\Delta}}{2}\left(x-\frac{\alpha+2 \beta_{1} k-\beta_{2} \omega}{\beta_{2} k-1} t\right)\right)\right]^{-1}\right\} \mathrm{e}^{\mathrm{i}(-k x+\omega t+\theta)} .
\end{aligned}
$$

\section{The improved Bernoulli sub-ODE method}

In this section, the IBSOM is utilized to discover travelling wave solutions of nonlinear PDEs [30, 31]. We consider the following steps:

Step 1. Let us consider a general form of the nonlinear partial differential equation

$$
\mathcal{F}\left(u, u_{x}, u_{t}, u_{x x}, u_{t t}, \ldots\right)=0,
$$

where $F$ is a function of $u(x, t), u_{t}(x, t), u_{x}(x, t)$, $u_{x x}(x, t), \ldots$ including nonlinear terms, and the subscripts denote the partial derivatives.

The travelling wave variable transformation is set, that is, $u(x, t)=u(\xi), \xi=x-\nu t$, where $\nu$ is arbitrary constant. Then Eq. (25) is converted into an ODE as follows

$$
\mathcal{Q}\left(u, u^{\prime},-\nu u^{\prime}, u^{\prime \prime}, \nu^{2} u^{\prime \prime}, \ldots\right)=0,
$$

where $Q$ is a function of $u, \frac{\mathrm{d} u}{\mathrm{~d} \xi}, \frac{\mathrm{d}^{2} u}{\mathrm{~d} \xi^{2}}, \ldots$ with nonlinear terms.

Step 2. Let us consider the solution of Eq. (26) as

$$
\begin{aligned}
& u(\xi)=\frac{\sum_{k=0}^{n} a_{k} F^{k}(\xi)}{\sum_{k=0}^{m} b_{k} F^{k}(\xi)}= \\
& \frac{a_{0}+a_{1} F(\xi)+a_{2} F^{2}(\xi)+\ldots+a_{n} F^{n}(\xi)}{b_{0}+b_{1} F(\xi)+b_{2} F^{2}(\xi)+\ldots+b_{m} F^{m}(\xi)},
\end{aligned}
$$

and according to the Bernoulli theory,

$$
\begin{aligned}
& F^{\prime}(\xi)=\varepsilon F(\xi)+\delta F^{\theta}(\xi), \quad \varepsilon \neq 0, \quad \delta \neq 0, \\
& \theta \in \mathbb{R}-\{0,1,2\},
\end{aligned}
$$

where $F(\xi)$ is the Bernoulli differential polynomial. Putting the above relations in Eq. (26), we have an equation of polynomial $\Psi(F(\xi))$ of $F(\xi)$ :

$$
\Psi(F(\xi))=\rho_{s} F^{s}(\xi)+\ldots \rho_{1} F(\xi)+\rho_{0}=0 .
$$

According to the homogeneous balance method, we can obtain the relationship between $n, m$, and $\theta$.

Step 3. Let all the coefficients of $\Psi(F(\xi))$ be zero. Then it yields an equation system as follows:

$$
\rho_{k}=0, \quad k=0,1, \ldots, s .
$$

Solving this system, we will determine the values of $a_{0}, \ldots, a_{n}$ and $b_{0}, \ldots, b_{m}$.

Step 4. By solving nonlinear Bernoulli differential Eq. (28), we obtain two cases according to $\varepsilon$ and $\delta$ situations as follows:

$$
\begin{aligned}
& F(\xi)=\left[\frac{-\delta}{\varepsilon}+\frac{E}{\mathrm{e}^{\varepsilon(\theta-1) \xi}}\right]^{\frac{1}{1-\theta}}, \\
& \varepsilon \neq \delta, \\
& F(\xi)=\left[\frac{E-1+(E+1) \tanh \left(\frac{\varepsilon(1-\theta) \xi}{2}\right)}{1-\tanh \left(\frac{\varepsilon(1-\theta) \xi}{2}\right)}\right]^{\frac{1}{1-\theta}}, \\
& \varepsilon=\delta, \quad E \in \mathbb{R},
\end{aligned}
$$

where $E$ is the non-zero constant of integration. Using a complete discrimination system for polynomial of $F(\xi)$, we solve Eq. (30) with the help of Maple 13 and classify the exact solutions for Eq. (26). 


\subsection{Application of IBSOM}

Considering Eqs. (27) and (28) for the homogeneous balance method between $u^{3}$ and $u^{\prime \prime}$ in Eq. (3), we obtain the following relationship for $m, n$, and $\theta$ :

$$
\theta+m=n+1 \text {. }
$$

For different values of $\theta, m$, and $n$, we have the following cases:

Case I: $\theta=n=3, m=1$.

If we take $\theta=n=3$ and $m=1$ in Eq. (27), then we obtain

$u(\xi)=\frac{a_{0}+a_{1} F(\xi)+a_{2} F^{2}(\xi)+a_{3} F^{3}(\xi)}{b_{0}+b_{1} F(\xi)}=\frac{\Theta_{1}(\xi)}{\Phi_{1}(\xi)}$,

$$
\begin{aligned}
& u^{\prime}(\xi)=\frac{\Theta_{1}^{\prime}(\xi) \Phi_{1}(\xi)-\Theta_{1}(\xi) \Phi_{1}^{\prime}(\xi)}{\Phi_{1}^{2}(\xi)}, \\
& u^{\prime \prime}(\xi)=\frac{\Theta_{1}^{\prime \prime}(\xi) \Phi_{1}(\xi)-\Theta_{1}(\xi) \Phi_{1}^{\prime}(\xi)}{\Phi_{1}^{2}(\xi)} \\
& -\frac{\left[\Theta_{1}(\xi) \Phi_{1}^{\prime}(\xi)\right]^{\prime} \Phi_{1}^{2}(\xi)-2 \Theta_{1}(\xi)\left[\Phi_{1}^{\prime}(\xi)\right]^{2} \Phi_{1}(\xi)}{\Phi_{1}^{4}(\xi)},
\end{aligned}
$$

where $F^{\prime}=\varepsilon F+\delta F^{3}, \varepsilon \neq 0, \delta \neq 0, a_{3} \neq 0$, and $b_{1} \neq 0$. When we use Eqs. (34) and (36) in Eq. (3), we get a system of algebraic equations. Therefore, we attain a system of equations from the coefficients of polynomial of $F$. This system of equations is solved for the above parameters with the following cases of solutions:

\section{Subset I:}

$\delta=0, \quad k=\frac{b}{\lambda-\sigma}, \quad \omega=\frac{\epsilon^{2} \beta_{2}(\lambda-\sigma)^{2}\left(\alpha \lambda-\alpha \sigma+2 b \beta_{1}\right)+\left(b \beta_{2}-\lambda+\sigma\right)\left(\alpha \lambda^{2}-2 \alpha \lambda \sigma+\alpha \sigma^{2}+b^{2} \beta_{1}\right)}{(\lambda-\sigma)\left[\left(\epsilon^{2} \beta_{2}^{2}+1\right)(\lambda-\sigma)^{2}-2 b \beta_{2}(\lambda-\sigma)+b^{2} \beta_{2}^{2}\right]}$,

$a_{0}=-\frac{\left[\epsilon^{2} \beta_{2}^{2}(\lambda-\sigma)^{2}+\left(b \beta_{2}-\lambda+\sigma\right)^{2}\right] b_{0} \beta_{1}}{\left[b^{2} \beta_{1} \beta_{2}+(\lambda-\sigma)\left(\alpha b \beta_{2}-\alpha \lambda \beta_{2}+\alpha \sigma \beta_{2}-\alpha \lambda+\alpha \sigma-2 b \beta_{1}\right)\right] \epsilon^{2} \beta_{2}}, \quad a_{3}=0$,

$a_{1}=\frac{\epsilon^{2} a_{2} b_{0} \beta_{2}(\lambda-\sigma)\left(\alpha \beta_{2}(b-\lambda+\sigma)-2 b \beta_{1}\right)+\epsilon^{2} \beta_{1} \beta_{2}^{2}\left(b^{2} a_{2} b_{0}-b_{1}^{2}(\lambda-\sigma)^{2}\right)-\alpha \epsilon^{2} a_{2} b_{0} \beta_{2}(\lambda-\sigma)^{2}-b_{1}^{2} \beta_{1}\left(b \beta_{2}-\lambda+\sigma\right)^{2}}{\left[\alpha\left(\lambda^{2}+2 \lambda \sigma-\sigma^{2}\right)+(\lambda-\sigma)\left(\alpha b \beta_{2}-\alpha \lambda \beta_{2}+\alpha \sigma \beta_{2}-2 b \beta_{1}\right)+b^{2} \beta_{1} \beta_{2}\right] b_{1} \epsilon^{2} \beta_{2}}$.

Based on the (37) and (31) we have

$$
\begin{aligned}
& u_{1}(x, t)=\frac{a_{0}+a_{1}\left[\frac{E}{\exp \left(2 \varepsilon\left(x-\frac{\alpha+2 \beta_{1} k-\beta_{2} \omega}{\beta_{2} k-1} t\right)\right)}\right]^{-\frac{1}{2}}+a_{2}\left[\frac{E}{\exp \left(2 \varepsilon\left(x-\frac{\alpha+2 \beta_{1} k-\beta_{2} \omega}{\beta_{2} k-1} t\right)\right)}\right]^{-1}}{b_{0}+b_{1}\left[\frac{E}{\exp \left(2 \varepsilon\left(x-\frac{\alpha+2 \beta_{1} k-\beta_{2} \omega}{\beta_{2} k-1} t\right)\right)}\right]^{-\frac{1}{2}}}, \\
& \psi_{1}(x, t)=\frac{a_{0}+a_{1}\left[\frac{E}{\exp \left(2 \varepsilon\left(x-\frac{\alpha+2 \beta_{1} \frac{b}{\lambda-\sigma}-\beta_{2} \omega}{\beta_{2} \frac{b}{\lambda-\sigma}-1} t\right)\right)}\right]^{-\frac{1}{2}}+a_{2}\left[\frac{E}{\exp \left(2 \varepsilon\left(x-\frac{\alpha+2 \beta_{1} \frac{b}{\lambda-\sigma}-\beta_{2} \omega}{\beta_{2} \frac{b}{\lambda-\sigma}-1} t\right)\right)}\right]}{b_{0}+b_{1}\left[\frac{1}{\exp \left(2 \varepsilon\left(x-\frac{\alpha+2 \beta_{1} \frac{b}{\lambda-\sigma}-\beta_{2} \omega}{\beta_{2} \frac{b}{\lambda-\sigma}-1} t\right)\right)}\right]^{-1}} \\
& \quad \times \exp \left(-\frac{b}{\lambda-\sigma} x+\omega t+\theta\right) .
\end{aligned}
$$

\section{Subset II:}

$$
\begin{aligned}
& \delta=0, \quad k=\frac{b}{\lambda-\sigma}, \\
& \omega=\frac{\epsilon^{2} \beta_{2}(\lambda-\sigma)^{2}\left(\alpha \lambda-\alpha \sigma+2 b \beta_{1}\right)+\left(b \beta_{2}-\lambda+\sigma\right)\left(\alpha \lambda^{2}-2 \alpha \lambda \sigma+\alpha \sigma^{2}+b^{2} \beta_{1}\right)}{(\lambda-\sigma)\left[\left(\epsilon^{2} \beta_{2}^{2}+1\right)(\lambda-\sigma)^{2}-2 b \beta_{2}(\lambda-\sigma)+b^{2} \beta_{2}^{2}\right]}, \\
& a_{0}=-\frac{\left[\epsilon^{2} \beta_{2}^{2}(\lambda-\sigma)^{2}+\left(b \beta_{2}-\lambda+\sigma\right)^{2}\right] b_{0} \beta_{1}}{\left[b^{2} \beta_{1} \beta_{2}+(\lambda-\sigma)\left(\alpha b \beta_{2}-\alpha \lambda \beta_{2}+\alpha \sigma \beta_{2}-\alpha \lambda+\alpha \sigma-2 b \beta_{1}\right)\right] \epsilon^{2} \beta_{2}}, \quad a_{1}=0, \quad a_{3}=0, \\
& b_{1}= \pm \varepsilon \sqrt{\frac{(\lambda-\sigma)\left(\alpha b \beta_{2}-\alpha \lambda \beta_{2}+\alpha \sigma \beta_{2}-2 b \beta_{1}\right)-\alpha(\lambda-\sigma)^{2}+b^{2} \beta_{1} \beta_{2}}{\beta_{1}\left[\epsilon^{2} \beta_{2}^{2}(\lambda-\sigma)^{2}+\left(b \beta_{2}-\lambda+\sigma\right)^{2}\right]}} .
\end{aligned}
$$


Based on the (39) and (31) we have

$$
\begin{aligned}
u_{2}(x, t)= & \frac{a_{0}+a_{2}\left[\frac{E}{\exp \left(2 \varepsilon\left(x-\frac{\alpha+2 \beta_{1} k-\beta_{2} \omega}{\beta_{2} k-1} t\right)\right)}\right]^{-1}}{b_{0}+b_{1}\left[\frac{E}{\exp \left(2 \varepsilon\left(x-\frac{\alpha+2 \beta_{1} k-\beta_{2} \omega}{\beta_{2} k-1} t\right)\right)}\right]^{-\frac{1}{2}}}, \\
\psi_{2}(x, t)= & \frac{a_{0}+a_{2}\left[\frac{E}{\exp \left(2 \varepsilon\left(x-\frac{\alpha+2 \beta_{1} k-\beta_{2} \omega}{\beta_{2} k-1}\right)\right)}\right]^{-1}}{b_{0}+b_{1}\left[\frac{E}{\exp \left(2 \varepsilon\left(x-\frac{\alpha+2 \beta_{1} k-\beta_{2} \omega}{\beta_{2} k-1}\right)\right)}\right]^{-\frac{1}{2}}} \exp \left(-\frac{b}{\lambda-\sigma} x+\omega t+\theta\right) .
\end{aligned}
$$

\section{Subset III:}

$\delta=0, \quad k=\frac{b}{\lambda-\sigma}, \quad \omega=\frac{b^{3} \beta_{1} b e t a_{2}-\alpha(\lambda-\sigma)^{3}+4 \epsilon^{2} \beta_{2}(\lambda-\sigma)^{2}\left(\alpha \lambda-\alpha \sigma+2 b \beta_{1}\right)+b(\lambda-\sigma)\left(\alpha \lambda \beta_{2}-\alpha \sigma \beta_{2}-b \beta_{1}\right)}{(\lambda-\sigma)\left[4 \epsilon^{2} \beta_{2}^{2}(\lambda-\sigma)^{2}+\left(b \beta_{2}-\lambda+\sigma\right)^{2}\right]}$, $b_{1}=\frac{a_{3} b_{0}}{a_{2}}, \quad a_{0}=-\frac{\left[4 \epsilon^{2} \beta_{2}^{2}(\lambda-\sigma)^{2}+\left(b \beta_{2}-\lambda+\sigma\right)^{2}\right] b_{0} \beta_{1}}{4\left[b^{2} \beta_{1} \beta_{2}+(\lambda-\sigma)\left(\alpha b \beta_{2}-\alpha \lambda \beta_{2}+\alpha \sigma \beta_{2}-\alpha \lambda+\alpha \sigma-2 b \beta_{1}\right)\right] \epsilon^{2} \beta_{2}}$,

$a_{1}=-\frac{\left[4 \epsilon^{2} \beta_{2}^{2}(\lambda-\sigma)^{2}+\left(b \beta_{2}-\lambda+\sigma\right)^{2}\right] b_{0} \beta_{1}}{4\left[b^{2} \beta_{1} \beta_{2}+(\lambda-\sigma)\left(\alpha b \beta_{2}-\alpha \lambda \beta_{2}+\alpha \sigma \beta_{2}-\alpha \lambda+\alpha \sigma-2 b \beta_{1}\right)\right] a_{2} \epsilon^{2} \beta_{2}}$,

Based on the (41) and (31) we have

$$
\begin{aligned}
& u_{3}(x, t)=\frac{a_{0}+a_{1}\left[\frac{E}{\exp \left(2 \varepsilon\left(x-\frac{\alpha+2 \beta_{1} k-\beta_{2} \omega}{\beta_{2} k-1} t\right)\right)}\right]^{-\frac{1}{2}}+a_{2}\left[\frac{E}{\exp \left(2 \varepsilon\left(x-\frac{\alpha+2 \beta_{1} k-\beta_{2} \omega}{\beta_{2} k-1} t\right)\right)}\right]^{-1}+a_{3}\left[\frac{E}{\exp \left(2 \varepsilon\left(x-\frac{\alpha+2 \beta_{1} k-\beta_{2} \omega}{\beta_{2} k-1} t\right)\right)}\right]^{-\frac{3}{2}}}{]^{-\frac{1}{2}}}, \\
& b_{0}+b_{1}\left[\frac{E}{\exp \left(2 \varepsilon\left(x-\frac{\alpha+2 \beta_{1} k-\beta_{2} \omega}{\beta_{2} k-1} t\right)\right)}\right]^{-\frac{1}{2}} \\
& \psi_{3}(0 x, t)=\frac{a_{0}+a_{1}\left[\frac{E}{\exp \left(2 \varepsilon\left(x-\frac{\alpha+2 \beta_{1} k-\beta_{2} \omega}{\beta_{2} k-1} t\right)\right)}\right]^{-\frac{1}{2}}+a_{2}\left[\frac{E}{\exp \left(2 \varepsilon\left(x-\frac{\alpha+2 \beta_{1} k-\beta_{2} \omega}{\beta_{2} k-1} t\right)\right)}\right]^{-1}+a_{3}\left[\frac{E}{\exp \left(2 \varepsilon\left(x-\frac{\alpha+2 \beta_{1} k-\beta_{2} \omega}{\beta_{2} k-1} t\right)\right)}\right]^{-\frac{3}{2}}}{b_{0}+b_{1}\left[\frac{E}{\exp \left(2 \varepsilon\left(x-\frac{\alpha+2 \beta_{1} k-\beta_{2} \omega}{\beta_{2} k-1} t\right)\right)}\right]^{-\frac{1}{2}}} \\
& \times \exp \left(-\frac{b}{\lambda-\sigma} x+\omega t+\theta\right) .
\end{aligned}
$$

Case II: $\theta=3, n=2$, and $m=0$.

If we take $\theta=3, n=2$, and $m=0$ in Eq. (1), then we obtain

$$
\begin{aligned}
& U(\xi)=\frac{a_{0}+a_{1} F(\xi)+a_{2} F^{2}(\xi)}{b_{0}}=\frac{\Theta_{2}(\xi)}{\Phi_{2}(\xi)}, \\
& U^{\prime}(\xi)=\frac{\Theta_{2}^{\prime}(\xi) \Phi_{2}(\xi)-\Theta_{2}(\xi) \Phi_{2}^{\prime}(\xi)}{\Phi_{2}^{2}(\xi)}, \\
& U^{\prime \prime}(\xi)=\frac{\Theta_{2}^{\prime \prime}(\xi) \Phi_{2}(\xi)-\Theta_{2}(\xi) \Phi_{2}^{\prime}(\xi)}{\Phi_{2}^{2}(\xi)}
\end{aligned}
$$

\section{Subset I:}

$\delta=0, \quad k=\frac{b}{\lambda-\sigma}, \quad \omega=\frac{b^{3} \beta_{1} \text { beta }_{2}-\alpha(\lambda-\sigma)^{3}+4 \epsilon^{2} \beta_{2}(\lambda-\sigma)^{2}\left(\alpha \lambda-\alpha \sigma+2 b \beta_{1}\right)+b(\lambda-\sigma)\left(\alpha \lambda \beta_{2}-\alpha \sigma \beta_{2}-b \beta_{1}\right)}{(\lambda-\sigma)\left[4 \epsilon^{2} \beta_{2}^{2}(\lambda-\sigma)^{2}+\left(b \beta_{2}-\lambda+\sigma\right)^{2}\right]}$,

$a_{1}=0, \quad b_{0}=-\frac{4 a_{0} \epsilon^{2} \beta_{2}\left[b^{2} \beta_{1} \beta_{2}+(\lambda-\sigma)\left(\alpha b \beta_{2}-\alpha \lambda \beta_{2}+\alpha \sigma \beta_{2}-2 b \beta_{1}\right)-\alpha(\lambda-\sigma)^{2}\right]}{\left[4 \epsilon^{2} \beta_{2}^{2}(\lambda-\sigma)^{2}+\left(b \beta_{2}-\lambda+\sigma\right)^{2}\right] \beta_{1}}$. 
Based on the (46) and (31) we have

$$
\begin{aligned}
& u_{4}(x, t)=\frac{a_{0}}{b_{0}}+\frac{a_{2}}{b_{0}}\left[\frac{E}{\exp \left(2 \varepsilon\left(x-\frac{\alpha+2 \beta_{1} \frac{b}{\lambda-\sigma}-\beta_{2} \omega}{\beta_{2} \frac{b}{\lambda-\sigma}-1} t\right)\right)}\right]^{-1}, \\
& \psi_{4}(x, t)= \\
& \left\{\frac{a_{0}}{b_{0}}+\frac{a_{2}}{b_{0}}\left[\frac{E}{\exp \left(2 \varepsilon\left(x-\frac{\alpha+2 \beta_{1} \frac{b}{\lambda-\sigma}-\beta_{2} \omega}{\beta_{2} \frac{b}{\lambda-\sigma}-1} t\right)\right)}\right]^{-1}\right\} \\
& \quad \times \exp \left(-\frac{b}{\lambda-\sigma} x+\omega t+\theta\right) .
\end{aligned}
$$

Case III: $\theta=3, n=4, m=2$.

If we take $\theta=3, n=4$ and $m=2$ in Eq. (27), then we obtain

$$
\begin{gathered}
u(\xi)=\frac{a_{0}+a_{1} F(\xi)+a_{2} F^{2}(\xi)+a_{3} F^{3}(\xi)+a_{4} F^{4}(\xi)}{b_{0}+b_{1} F(\xi)+b_{2} F^{2}(\xi)}= \\
\frac{\Theta_{3}(\xi)}{\Phi_{3}(\xi)}, \\
u^{\prime \prime}(\xi)=\frac{\Theta_{3}^{\prime \prime}(\xi) \Phi_{3}(\xi)-\Theta_{3}(\xi) \Phi_{3}^{\prime}(\xi)}{\Phi_{3}^{2}(\xi)} \\
-\frac{\left[\Theta_{3}(\xi) \Phi_{3}^{\prime}(\xi)\right]^{\prime} \Phi_{3}^{2}(\xi)-2 \Theta_{3}(\xi)\left[\Phi_{3}^{\prime}(\xi)\right]^{2} \Phi_{3}(\xi)}{\Phi_{3}^{4}(\xi)},
\end{gathered}
$$

where $F^{\prime}=\varepsilon F+\delta F^{3}, \varepsilon \neq 0, \delta \neq 0, a_{4} \neq 0$, and $b_{2} \neq 0$. When we use Eqs. (48) and (49) in Eq. (3), we get a system of algebraic equations. Therefore, we attain a system of equations from the coefficients of polynomial of $F$. This system of equations is solved for the above parameters with the following cases of solutions:

\section{Subset I}

$\varepsilon=\left(b \beta_{2}-\lambda+\sigma\right) b_{2} \sqrt{-\frac{\beta_{1}}{\Omega}}, \quad \delta=0, \quad k=\frac{b}{\lambda-\sigma}, \quad \omega=\frac{\left(a_{2} b_{2}-a_{4} b_{0}\right)\left(\alpha \lambda^{2}-2 \alpha \lambda \sigma+\alpha \sigma^{2}+b^{2} \beta_{1}\right)-b_{2}^{2} \beta_{1}(\lambda-\sigma)^{2}}{\left(a_{2} b_{2}-a_{4} b_{0}\right)(\lambda-\sigma)\left(b \beta_{2}-\lambda+\sigma\right)}$, $\Omega=4 \beta_{1} \beta_{2}^{2}\left(b^{2} a_{2} b_{2}-b^{2} a_{4} b_{0}+\lambda^{2} b_{2}^{2}-2 \lambda \sigma b_{2}^{2}+\sigma^{2} b_{2}^{2}\right)+4 \beta_{2}\left(a_{2} b_{2}-a_{4} b_{0}\right)(\lambda-\sigma)\left(\alpha b \beta_{2}-\alpha \lambda \beta_{2}+\alpha \sigma \beta_{2}-\alpha \lambda+\alpha \sigma-2 b \beta_{1}\right)$,

$a_{0}=\frac{b_{0}\left(a_{2} b_{2}-a_{4} b_{0}\right)}{b_{2}^{2}}, \quad a_{3}=\frac{a_{1} a_{4} b_{2}}{a_{2} b_{2}-a_{4} b_{0}}, \quad b_{1}=\frac{a_{1} b_{2}^{2}}{a_{2} b_{2}-a_{4} b_{0}},$.

Based on the (50) and (31) we have

$$
\begin{aligned}
& u_{5}(x, t)=\frac{\frac{b_{0}\left(a_{2} b_{2}-a_{4} b_{0}\right)}{b_{2}^{2}}+a_{1}\left[\frac{E}{\mathrm{e}^{2 \varepsilon(x-\nu t)}}\right]^{-\frac{1}{2}}+a_{2}\left[\frac{E}{\mathrm{e}^{2 \varepsilon(x-\nu t)}}\right]^{-1}+\frac{a_{1} a_{4} b_{2}}{a_{2} b_{2}-a_{4} b_{0}}\left[\frac{E}{\mathrm{e}^{2 \varepsilon(x-\nu t)}}\right]^{-\frac{3}{2}}+a_{4}\left[\frac{E}{\mathrm{e}^{2 \varepsilon(x-\nu t)}}\right]^{-2}}{b_{0}+\frac{a_{1} b_{2}^{2}}{a_{2} b_{2}-a_{4} b_{0}}\left[\frac{E}{\mathrm{e}^{2 \varepsilon(x-\nu t)}}\right]^{-\frac{1}{2}}+b_{2}\left[\frac{E}{\mathrm{e}^{2 \varepsilon(x-\nu t)}}\right]^{-1}}, \\
& \psi_{5}(x, t)=\frac{\frac{b_{0}\left(a_{2} b_{2}-a_{4} b_{0}\right)}{b_{2}^{2}}+a_{1}\left[\frac{E}{\mathrm{e}^{2 \varepsilon(x-\nu t)}}\right]^{-\frac{1}{2}}+a_{2}\left[\frac{E}{\mathrm{e}^{2 \varepsilon(x-\nu t)}}\right]^{-1}+\frac{a_{1} a_{4} b_{2}}{a_{2} b_{2}-a_{4} b_{0}}\left[\frac{E}{\mathrm{e}^{2 \varepsilon(x-\nu t)}}\right]^{\frac{-3}{2}}+a_{4}\left[\frac{E}{\mathrm{e}^{2 \varepsilon(x-\nu t)}}\right]^{-2}}{b_{0}+\frac{a_{1} b_{2}^{2}}{a_{2} b_{2}-a_{4} b_{0}}\left[\frac{E}{\mathrm{e}^{2 \varepsilon(x-\nu t)}}\right]^{-\frac{1}{2}}+b_{2}\left[\frac{E}{\mathrm{e}^{2 \varepsilon(x-\nu t)}}\right]^{-1}} \\
& \quad \times \exp \left(-\frac{b}{\lambda-\sigma} x+\omega t+\theta\right),
\end{aligned}
$$

where $\nu=\frac{\alpha+2 \beta_{1} \frac{b}{\lambda-\sigma}-\beta_{2} \omega}{\beta_{2} \frac{b}{\lambda-\sigma}-1}$.

\section{Description of the GGM}

The following steps of the generalized $\left(\mathrm{G}^{\prime} / \mathrm{G}\right)$ expansion method [32] are considered as:

Step 1. Let us consider a general form of the nonlinear partial differential equation

$$
\mathcal{F}\left(u, u_{x}, u_{t}, u_{x x}, u_{t t}, \ldots\right)=0,
$$

where $F$ is a function of $u(x, t), u_{t}(x, t), u_{x}(x, t)$, $u_{x x}(x, t), \ldots$ including nonlinear terms, and the subscripts denote the partial derivatives.

The travelling wave variable transformation is set, that is, $u(x, t)=u(\xi), \xi=x-\nu t$, where $\nu$ is arbitrary constant. Then Eq. (25) is converted into an ODE as follows:

$$
\mathcal{Q}\left(u, u^{\prime},-\nu u^{\prime}, u^{\prime \prime}, \nu^{2} u^{\prime \prime}, \ldots\right)=0,
$$

where $Q$ is a function of $u, \frac{\mathrm{d} u}{\mathrm{~d} \xi}, \frac{\mathrm{d}^{2} u}{\mathrm{~d} \xi^{2}}, \ldots$ with nonlinear terms.

Step 2. Let us consider the solution of Eq. (53) as

$$
u(\xi)=S(\Phi(\xi))=\sum_{k=0}^{m} A_{k} \Phi(\xi)^{k}+\sum_{k=1}^{m} B_{k} \Phi(\xi)^{-k}
$$

where $d_{k}(0 \leq k \leq m)$ are constants to be determined, such that $d_{m} \neq 0, e_{m} \neq 0$ and $\Phi(\xi)=G^{\prime}(\xi) / G(\xi)$ satisfies the following ordinary differential equation:

$$
k_{1} G G^{\prime \prime}-k_{2} G G^{\prime}-k_{3}\left(G^{\prime}\right)^{2}-k_{4} G^{2}=0 .
$$


We will consider the special solutions of Eq. (55):

Family 1: When $k_{2} \neq 0, f=k_{1}-k_{3}$ and $s=k_{2}^{2}+4 k_{4}\left(k_{1}-k_{3}\right)>0$, then

$$
\Phi(\xi)=\frac{k_{2}}{2 f}+\frac{\sqrt{s}}{2 f} \frac{C_{1} \sinh \left(\frac{\sqrt{s}}{2 k_{1}} \xi\right)+C_{2} \cosh \left(\frac{\sqrt{s}}{2 k_{1}} \xi\right)}{C_{1} \cosh \left(\frac{\sqrt{s}}{2 k_{1}} \xi\right)+C_{2} \sinh \left(\frac{\sqrt{s}}{2 k_{1}} \xi\right)} .
$$

Family 2: When $k_{2} \neq 0, f=k_{1}-k_{3}$ and $s=k_{2}^{2}+4 k_{4}\left(k_{1}-k_{3}\right)<0$, then

$$
\Phi(\xi)=\frac{k_{2}}{2 f}+\frac{\sqrt{-s}}{2 f} \frac{-C_{1} \sin \left(\frac{\sqrt{-s}}{2 k_{1}} \xi\right)+C_{2} \cos \left(\frac{\sqrt{-s}}{2 k_{1}} \xi\right)}{C_{1} \cos \left(\frac{\sqrt{-s}}{2 k_{1}} \xi\right)+C_{2} \sin \left(\frac{\sqrt{-s}}{2 k_{1}} \xi\right)} .
$$

Family 3: When $k_{2} \neq 0, f=k_{1}-k_{3}$ and $s=k_{2}^{2}+$ $4 k_{4}\left(k_{1}-k_{3}\right)=0$, then $\Phi(\xi)=\frac{k_{2}}{2 f}+\frac{C_{2}}{C_{1}+C_{2} \xi}$.

Family 4: When $k_{2}=0, f=k_{1}-k_{3}$ and $g=f k_{4}>0$, then

$$
\Phi(\xi)=\frac{\sqrt{g}}{f} \frac{C_{1} \sinh \left(\frac{\sqrt{g}}{k_{1}} \xi\right)+C_{2} \cosh \left(\frac{\sqrt{g}}{k_{1}} \xi\right)}{C_{1} \cosh \left(\frac{\sqrt{g}}{k_{1}} \xi\right)+C_{2} \sinh \left(\frac{\sqrt{g}}{k_{1}} \xi\right)} .
$$

Family 5: When $k_{2}=0, f=k_{1}-k_{3}$ and $g=f k_{4}<0$, then

$$
\Phi(\xi)=\frac{\sqrt{-g}}{f} \frac{-C_{1} \sin \left(\frac{\sqrt{-g}}{k_{1}} \xi\right)+C_{2} \cos \left(\frac{\sqrt{-g}}{k_{1}} \xi\right)}{C_{1} \cos \left(\frac{\sqrt{-g}}{k_{1}} \xi\right)+C_{2} \sin \left(\frac{\sqrt{-g}}{k_{1}} \xi\right)} .
$$

Family 6: When $k_{4}=0$ and $f=k_{1}-k_{3}$, then

$$
\Phi(\xi)=\frac{C_{1} k_{2}^{2} \exp \left(\frac{-k_{2}}{k_{1}} \xi\right)}{f k_{1}+C_{1} k_{1} k_{2} \exp \left(\frac{-k_{2}}{k_{1}} \xi\right)} .
$$

Family 7: When $k_{2} \neq 0$ and $f=k_{1}-k_{3}=0$, then $\Phi(\xi)=-\frac{k_{4}}{k_{2}}+C_{1} \exp \left(\frac{k_{2}}{k_{1}} \xi\right)$,
Family 8: When $k_{1}=k_{3}, k_{2}=0$ and $f=k_{1}-k_{3}=0$, then $\Phi(\xi)=C_{1}+\frac{k_{4}}{k_{1}} \xi$,

Family 9: When $k_{3}=2 k_{1}, k_{2}=0$ and $k_{4}=0$, then

$$
\Phi(\xi)=-\frac{1}{C_{1}+\left(\frac{k_{3}}{k_{1}}-1\right) \xi},
$$

where $d_{0}, d_{j}, e_{j}(j=1, \ldots, m), k_{1}, k_{2}, k_{3}$, and $k_{4}$ are constants to be determined later. Although, the positive integer $m$ can be determined by considering the homogeneous balance between the highest order derivatives and nonlinear terms appearing in Eq. (55). If $m$ is not an integer, then a transformation formula should be used to overcome this difficulty.

Step 3. Substituting (54) into Eq. (53) with the value of $m$ obtained in Step 2, collecting the coefficients of $\Phi(\xi)^{k}, \Phi(\xi)^{-k}(k=0,1,2, \ldots)$, and then setting each coefficient to zero, we can get a set of over-determined equations for $A_{0}, A_{k}, B_{k}(k=1, \ldots, m), k_{1}, k_{2}, k_{3}, k_{4}$ with the aid of symbolic computation using Maple 13.

Step 4. We solve the algebraic equations in Step 3. We then substituting $A_{0}, A_{1}, B_{1}, \ldots, A_{m}, B_{m}, \nu$ in (54).

\subsection{Application of $G G M$}

By processing the generalized $G^{\prime} / G$-expansion method to Eq. (1) and by balancing the terms $u^{\prime \prime}$ and $u^{3}$ by using homogeneous principle, we get to

$$
m+2=3 m \Longrightarrow m=1
$$

The closed form solution is

$$
u(\xi)=d_{0}+d_{1} \Phi(\xi)+e_{1} \Phi(\xi) .
$$

Solving the nonlinear algebraic equations, we have the following sets of coefficients for the solutions of (57) as given below:

Subset I. We have the following

$k=\frac{b}{\lambda-\sigma}, \quad \omega=\frac{k_{1}^{2}\left(b \beta_{2}-\lambda+\sigma\right)\left(\alpha(\lambda-\sigma)^{2}+b^{2} \beta_{1}\right)+\beta_{2} k_{2}^{2}(\lambda-\sigma)^{2}\left(\alpha \lambda-\alpha \sigma+2 b \beta_{1}\right)}{(\lambda-\sigma)\left[k_{1}^{2}\left(b \beta_{2}-\lambda+\sigma\right)^{2}+\beta_{2}^{2} k_{2}^{2}(\lambda-\sigma)^{2}\right]}, \quad B_{1}=0, \quad k_{3}=k_{1}$,

$k_{4}=\frac{\beta_{1} k_{1}^{2}\left(b \beta_{2}-\lambda+\sigma\right)^{2}+A_{0} \beta_{2} k_{2}^{2}(\lambda-\sigma)\left(\alpha b \beta_{2}-\alpha \lambda \beta_{2}+\alpha \sigma \beta_{2}-\alpha \lambda+\alpha \sigma-2 b \beta_{1}\right)+\beta_{1} \beta_{2}^{2} k_{2}^{2}\left(b^{2} A_{0}+\lambda^{2}-2 \lambda \sigma+\sigma^{2}\right)}{\left[(\lambda-\sigma)\left(\alpha b \beta_{2}-\alpha \lambda \beta_{2}+\alpha \sigma \beta_{2}-2 b \beta_{1}\right)-\alpha(\lambda-\sigma)^{2}+b^{2} \beta_{1} \beta_{2}\right] A_{1} \beta_{2} k_{2}}$.

Based on the Family 7, the kink-singular exact solution can be written as

$$
\psi_{1}(x, t)=\left\{A_{0}+A_{1}\left[-\frac{k_{4}}{k_{2}}+C_{1} \exp \left(\frac{k_{2}}{k_{1}}\left(x-\frac{\alpha+2 \beta_{1} \frac{b}{\lambda-\sigma}-\beta_{2} \omega}{\beta_{2} \frac{b}{\lambda-\sigma}-1} t\right)\right]^{-1}\right\} \exp \left(-\frac{b}{\lambda-\sigma} x+\omega t+\theta\right) .\right.
$$

Based on the Family 8, the cupson exact solution can be written as

$$
\psi_{2}(x, t)=\left\{A_{0}+A_{1}\left[C_{1}+\frac{k_{4}}{k_{1}}\left(x-\frac{\alpha+2 \beta_{1} \frac{b}{\lambda-\sigma}-\beta_{2} \omega}{\beta_{2} \frac{b}{\lambda-\sigma}-1} t\right)\right]^{-1}\right\} \exp \left(-\frac{b}{\lambda-\sigma} x+\omega t+\theta\right) .
$$

Subset II. We have the following

$$
k=\frac{b}{\lambda-\sigma}, \quad \omega=\frac{k_{1}^{2}\left(b \beta_{2}-\lambda+\sigma\right)\left(\alpha(\lambda-\sigma)^{2}+b^{2} \beta_{1}\right)+\beta_{2} k_{2}^{2}(\lambda-\sigma)^{2}\left(\alpha \lambda-\alpha \sigma+2 b \beta_{1}\right)}{(\lambda-\sigma)\left[k_{1}^{2}\left(b \beta_{2}-\lambda+\sigma\right)^{2}+\beta_{2}^{2} k_{2}^{2}(\lambda-\sigma)^{2}\right]}, \quad A_{1}=0
$$




$$
\begin{aligned}
& k_{3}=\frac{\beta_{1} \beta_{2}^{2} \Omega_{1}-2 b \beta_{1} \beta_{2} \Omega_{2}+\alpha \beta_{2}^{2} k_{2} \Omega_{3}-(\lambda-\sigma)^{2} \Omega_{4}}{\Omega_{5} B_{1} \beta_{2} k_{2}}, \quad k_{4}=0, \\
& \Omega_{1}=b^{2} A_{0} k_{2}^{2}+b^{2} B_{1} k_{1} k_{2}+b^{2} k_{1}^{2}+\lambda^{2} k_{2}^{2}-2 \lambda \sigma k_{2}^{2}+\sigma^{2} k_{2}^{2}, \quad \Omega_{2}=\left(A_{0} k_{2}^{2}+B_{1} k_{1} k_{2}+k_{1}^{2}\right)(\lambda-\sigma), \\
& \Omega_{3}=\left(A_{0} k_{2}+B_{1} k_{1}\right)(\lambda-\sigma)(b-\lambda+\sigma), \quad \Omega_{4}=\alpha A_{0} \beta_{2} k_{2}^{2}+\alpha B_{1} \beta_{2} k_{1} k_{2}-\beta_{1} k_{1}^{2}, \\
& \Omega_{5}=\left[\alpha \beta_{2}(\lambda-\sigma)(b-\lambda+\sigma)+b \beta_{1}\left(b \beta_{2}-2 \lambda+2 \sigma\right)-\alpha(\lambda-\sigma)^{2}\right] .
\end{aligned}
$$

Based on the Family 1, the exact hyperbolic solution can be written as

$$
\begin{aligned}
& \psi_{3}(x, t)=\exp \left(-\frac{b}{\lambda-\sigma} x+\omega t+\theta\right) \\
& \quad \times\left\{A_{0}+A_{1}\left[\frac{k_{2}}{2\left(k_{1}-k_{3}\right)}+\frac{k_{2}}{2\left(k_{1}-k_{3}\right)} \frac{C_{1} \sinh \left(\frac{k_{2}}{2 k_{1}}\left(x-\frac{\alpha+2 \beta_{1} \frac{b}{\lambda-\sigma}-\beta_{2} \omega}{\beta_{2} \frac{b}{\lambda-\sigma}-1} t\right)\right)+C_{2} \cosh \left(\frac{k_{2}}{2 k_{1}}\left(x-\frac{\alpha+2 \beta_{1} \frac{b}{\lambda-\sigma}-\beta_{2} \omega}{\beta_{2} \frac{b}{\lambda-\sigma}-1} t\right)\right)}{C_{1} \cosh \left(\frac{k_{2}}{2 k_{1}}\left(x-\frac{\alpha+2 \beta_{1} \frac{b}{\lambda-\sigma}-\beta_{2} \omega}{\beta_{2} \frac{b}{\lambda-\sigma}-1} t\right)\right)+C_{2} \sinh \left(\frac{k_{2}}{2 k_{1}}\left(x-\frac{\alpha+2 \beta_{1} \frac{b}{\lambda-\sigma}-\beta_{2} \omega}{\beta_{2} \frac{b}{\lambda-\sigma}-1} t\right)\right)}\right] .\right.
\end{aligned}
$$

Based on the Family 6, the exact kink-singular solution can be written as

$$
\psi_{4}(x, t)=\left\{A_{0}+A_{1}\left[\frac{C_{1} k_{2}^{2} \exp \left(\frac{-k_{2}}{k_{1}}\left(x-\frac{\alpha+2 \beta_{1} \frac{b}{\lambda-\sigma}-\beta_{2} \omega}{\beta_{2} \frac{b}{\lambda-\sigma}-1} t\right)\right)}{\left(k_{1}-k_{3}\right) k_{1}+C_{1} k_{1} k_{2} \exp \left(\frac{-k_{2}}{k_{1}}\left(x-\frac{\alpha+2 \beta_{1} \frac{b}{\lambda-\sigma}-\beta_{2} \omega}{\beta_{2} \frac{b}{\lambda-\sigma}-1} t\right)\right)}\right] \exp \left(-\frac{b}{\lambda-\sigma} x+\omega t+\theta\right) .\right.
$$

Subset III. We have the following

$$
\begin{aligned}
& k=\frac{b}{\lambda-\sigma}, \quad \omega=\frac{k_{1}^{2}\left(b \beta_{2}-\lambda+\sigma\right)\left(\alpha(\lambda-\sigma)^{2}+b^{2} \beta_{1}\right)+\beta_{2} k_{2}^{2}(\lambda-\sigma)^{2}\left(\alpha \lambda-\alpha \sigma+2 b \beta_{1}\right)}{(\lambda-\sigma)\left[k_{1}^{2}\left(b \beta_{2}-\lambda+\sigma\right)^{2}+\beta_{2}^{2} k_{2}^{2}(\lambda-\sigma)^{2}\right]}, \\
& k_{3}=k_{1}, \quad k_{4}=0, \quad A_{0}=-\frac{k_{1}^{2}\left(b \beta_{2}-\lambda+\sigma\right)^{2}+\beta_{2}^{2} k_{2}^{2}(\lambda-\sigma)^{2}}{\left[b \beta_{1}\left(b \beta_{2}-2 \lambda+2 \sigma\right)+\alpha \beta_{2}(\lambda-\sigma)(b-\lambda+\sigma)-\alpha(\lambda-\sigma)^{2}\right] \beta_{2} k_{2}^{2}} .
\end{aligned}
$$

Based on the Family 7, the exact kink solution can be written as

$$
\begin{aligned}
& \psi_{5}(x, t)=\exp \left(-\frac{b}{\lambda-\sigma} x+\omega t+\theta\right) \\
& \quad \times\left\{A_{0}+A_{1}\left[C_{1} \exp \left(\frac{k_{2}}{k_{1}}\left(x-\frac{\alpha+2 \beta_{1} \frac{b}{\lambda-\sigma}-\beta_{2} \omega}{\beta_{2} \frac{b}{\lambda-\sigma}-1} t\right)\right)+\frac{B_{1}}{C_{1} \exp \left(\frac{k_{2}}{k_{1}}\left(x-\frac{\alpha+2 \beta_{1} \frac{b}{\lambda-\sigma}-\beta_{2} \omega}{\beta_{2} \frac{b}{\lambda-\sigma}-1} t\right)\right)}\right]\right\} .
\end{aligned}
$$

Subset IV. We have the following

$$
\begin{aligned}
& \omega=\frac{A_{0}\left(k^{2} \beta_{1}+\alpha\right)-A_{0}^{3}(-k \lambda+k \sigma+b)+\beta_{1}}{\left(k \beta_{2}-1\right) A_{0}}, \quad k_{2}=\frac{2 A_{0}\left(k \beta_{2}-1\right) k_{1}}{\Omega_{1}}, \\
& k_{3}=\frac{k_{1}\left[4 \beta_{2} k_{4}\left(k^{2} A_{0} \beta_{1} \beta_{2}-\beta_{1} \beta_{2}-2 k A_{0} \beta_{1}-k \lambda A_{0}^{3} \beta_{2}+k \sigma A_{0}^{3} \beta_{2}+b A_{0}^{3} \beta_{2}+\alpha k A_{0} \beta_{2}-\alpha A_{0} \beta_{2}-\alpha A_{0}\right)-\beta_{1} k_{1}\left(k \beta_{2}-1\right)^{2}\right]}{4 \beta_{2} k_{4}\left[\beta_{1} \beta_{2}\left(k^{2} A_{0}-1\right)+A_{0}^{3} \beta_{2}(-k \lambda+k \sigma+b)+A_{0}\left(\alpha k \beta_{2}-\alpha \beta_{2}-2 k \beta_{1}-\alpha\right)\right]}, \\
& A_{1}=-\frac{\left(k \beta_{2}-1\right) \beta_{1} k_{1} \Omega_{1}}{4 \beta_{2} k_{4}\left[\beta_{1} \beta_{2}\left(k^{2} A_{0}-1\right)+A_{0}^{3} \beta_{2}(-k \lambda+k \sigma+b)+A_{0}\left(\alpha k \beta_{2}-\alpha \beta_{2}-2 k \beta_{1}-\alpha\right)\right]}, \quad B_{1}=\frac{k_{4} \Omega_{1}}{\left(k \beta_{2}-1\right) k_{1}}, \\
& \Omega_{1}=\sqrt{\frac{2 \alpha A_{0} \beta_{2}-2 A_{0} \beta_{2}^{2}\left(-k \lambda A_{0}^{2}+k \sigma A_{0}^{2}+b A_{0}^{2}+\alpha k-\alpha\right)-2 \beta_{1} \beta_{2}\left(k^{2} A_{0} \beta_{2}-2 k A_{0}-\beta_{2}\right)}{A_{0}(-k \lambda+k \sigma+b)}} .
\end{aligned}
$$

Based on the Family 1, the exact combined hyperbolic solution can be written as

$\psi_{6}(x, t)=\exp \left(-k x+\frac{A_{0}\left(k^{2} \beta_{1}+\alpha\right)-A_{0}^{3}(-k \lambda+k \sigma+b)+\beta_{1}}{\left(k \beta_{2}-1\right) A_{0}} t+\theta\right) \times$ 


$$
\begin{aligned}
& \times\left\{A_{0}+A_{1}\left[\frac{k_{2}}{2\left(k_{1}-k_{3}\right)}+\frac{\sqrt{s}}{2\left(k_{1}-k_{3}\right)} \frac{C_{1} \sinh \left(\frac{\sqrt{s}}{2 k_{1}}\left(x-\frac{\alpha+2 \beta_{1} k-\beta_{2} \omega}{\beta_{2} k-1} t\right)\right)+C_{2} \cosh \left(\frac{\sqrt{s}}{2 k_{1}}\left(x-\frac{\alpha+2 \beta_{1} k-\beta_{2} \omega}{\beta_{2} k-1} t\right)\right)}{C_{1} \cosh \left(\frac{\sqrt{s}}{2 k_{1}}\left(x-\frac{\alpha+2 \beta_{1} k-\beta_{2} \omega}{\beta_{2} k-1} t\right)\right)+C_{2} \sinh \left(\frac{\sqrt{s}}{2 k_{1}}\left(x-\frac{\alpha+2 \beta_{1} k-\beta_{2} \omega}{\beta_{2} k-1} t\right)\right)}\right]\right. \\
& \left.+\frac{k_{4} \Omega_{1}}{\left(k \beta_{2}-1\right) k_{1}}\left[\frac{k_{2}}{2\left(k_{1}-k_{3}\right)}+\frac{\sqrt{s}}{2\left(k_{1}-k_{3}\right)} \frac{C_{1} \sinh \left(\frac{\sqrt{s}}{2 k_{1}}\left(x-\frac{\alpha+2 \beta_{1} k-\beta_{2} \omega}{\beta_{2} k-1} t\right)\right)+C_{2} \cosh \left(\frac{\sqrt{s}}{2 k_{1}}\left(x-\frac{\alpha+2 \beta_{1} k-\beta_{2} \omega}{\beta_{2} k-1} t\right)\right)}{C_{1} \cosh \left(\frac{\sqrt{s}}{2 k_{1}}\left(x-\frac{\alpha+2 \beta_{1} k-\beta_{2} \omega}{\beta_{2} k-1} t\right)\right)+C_{2} \sinh \left(\frac{\sqrt{s}}{2 k_{1}}\left(x-\frac{\alpha+2 \beta_{1} k-\beta_{2} \omega}{\beta_{2} k-1} t\right)\right)}\right]^{-1}\right\},
\end{aligned}
$$

when

$$
s=\frac{\left(k^{2} \beta_{2}^{2}-2 k \beta_{2}+1\right) k_{1}^{2}\left(-2 k \lambda A_{0}^{3}+2 k \sigma A_{0}^{3}+2 b A_{0}^{3}+\beta_{1}\right)}{\beta_{2}\left[A_{0}^{3} \beta_{2}(-k \lambda+k \sigma+b)+A_{0}\left(k^{2} \beta_{1} \beta_{2}+\alpha k \beta_{2}-\alpha \beta_{2}-2 k \beta_{1}-\alpha\right)-\beta_{1} \beta_{2}\right]}>0 .
$$

Based on the Family 2, the exact solution can be written as

$$
\begin{aligned}
& \psi_{7}(x, t)=\exp \left(-k x+\frac{A_{0}\left(k^{2} \beta_{1}+\alpha\right)-A_{0}^{3}(-k \lambda+k \sigma+b)+\beta_{1}}{\left(k \beta_{2}-1\right) A_{0}} t+\theta\right) \\
& \times\left\{A_{0}+A_{1}\left[\frac{k_{2}}{2\left(k_{1}-k_{3}\right)}+\frac{\sqrt{-s}}{2\left(k_{1}-k_{3}\right)} \frac{-C_{1} \sin \left(\frac{\sqrt{-s}}{2 k_{1}}\left(x-\frac{\alpha+2 \beta_{1} k-\beta_{2} \omega}{\beta_{2} k-1} t\right)\right)+C_{2} \cos \left(\frac{\sqrt{-s}}{2 k_{1}}\left(x-\frac{\alpha+2 \beta_{1} k-\beta_{2} \omega}{\beta_{2} k-1} t\right)\right)}{C_{1} \cos \left(\frac{\sqrt{-s}}{2 k_{1}}\left(x-\frac{\alpha+2 \beta_{1} k-\beta_{2} \omega}{\beta_{2} k-1} t\right)\right)+C_{2} \sin \left(\frac{\sqrt{-s}}{2 k_{1}}\left(x-\frac{\alpha+2 \beta_{1} k-\beta_{2} \omega}{\beta_{2} k-1} t\right)\right)}\right] \quad(68)\right. \\
& \left.+\frac{k_{4} \Omega_{1}}{\left(k \beta_{2}-1\right) k_{1}}\left[\frac{k_{2}}{2\left(k_{1}-k_{3}\right)}+\frac{\sqrt{-s}}{2\left(k_{1}-k_{3}\right)} \frac{-C_{1} \sin \left(\frac{\sqrt{-s}}{2 k_{1}}\left(x-\frac{\alpha+2 \beta_{1} k-\beta_{2} \omega}{\beta_{2} k-1} t\right)\right)+C_{2} \cos \left(\frac{\sqrt{-s}}{2 k_{1}}\left(x-\frac{\alpha+2 \beta_{1} k-\beta_{2} \omega}{\beta_{2} k-1} t\right)\right)}{C_{1} \cos \left(\frac{\sqrt{-s}}{2 k_{1}}\left(x-\frac{\alpha+2 \beta_{1} k-\beta_{2} \omega}{\beta_{2} k-1} t\right)\right)+C_{2} \sin \left(\frac{\sqrt{-s}}{2 k_{1}}\left(x-\frac{\alpha+2 \beta_{1} k-\beta_{2} \omega}{\beta_{2} k-1} t\right)\right)}\right]^{-1}\right\},
\end{aligned}
$$

when

$$
s=\frac{\left(k^{2} \beta_{2}^{2}-2 k \beta_{2}+1\right) k_{1}^{2}\left(-2 k \lambda A_{0}^{3}+2 k \sigma A_{0}^{3}+2 b A_{0}^{3}+\beta_{1}\right)}{\beta_{2}\left[A_{0}^{3} \beta_{2}(-k \lambda+k \sigma+b)+A_{0}\left(k^{2} \beta_{1} \beta_{2}+\alpha k \beta_{2}-\alpha \beta_{2}-2 k \beta_{1}-\alpha\right)-\beta_{1} \beta_{2}\right]}<0 .
$$

Based on the Family 3 , the exact combined periodic solution can be written as

$$
\begin{aligned}
& \psi_{8}(x, t)=\exp \left(-k x+\frac{A_{0}\left(k^{2} \beta_{1}+\alpha\right)-A_{0}^{3}(-k \lambda+k \sigma+b)+\beta_{1}}{\left(k \beta_{2}-1\right) A_{0}} t+\theta\right) \\
& \times\left\{A_{0}+A_{1}\left[\frac{k_{2}}{2 f}+\frac{C_{2}}{C_{1}+C_{2}\left(x-\frac{\alpha+2 \beta_{1} k-\beta_{2} \omega}{\beta_{2} k-1}\right)}\right]+\frac{k_{4} \Omega_{1}}{\left(k \beta_{2}-1\right) k_{1}}\left[\frac{k_{2}}{2 f}+\frac{C_{2}}{C_{1}+C_{2}\left(x-\frac{\alpha+2 \beta_{1} k-\beta_{2} \omega}{\beta_{2} k-1}\right)}\right]^{-1}\right\},
\end{aligned}
$$

when $\beta_{1}=2 A_{0}^{3}(k \lambda-k \sigma-b)$.

\section{Conclusion}

In this paper, a FLE is studied in birefringent optical fibers with the aid of improved $\tan (\phi(\xi) / 2)$-expansion method, improved Bernoulli sub-ODE method and generalized $\left(G^{\prime} / G\right)$-expansion method using three different trial equations, to retrieve bright, dark, singular, and combo soliton solutions. The existence of these solitons are guaranteed by the constraint conditions on the parameters. The results of this paper are very useful for telecommunication industry in general and in fiber optics in particular. Moreover, in near future, the application of this integration tool will be used to solve other models that include optical couplers, FLE applied to DWDM systems, metasurfaces, and metamaterials.

\section{Acknowledgments}

This paper is published as part of a research project supported by the University of Tabriz Research Affairs Office.

\section{References}

[1] M.S. Osman, J.A.T. Machado, D. Baleanu, Opt. Quant. Electron. 50, 1 (2018).

[2] A. Biswas, Y. Yildirim, E. Yasar, Q. Zhou, M.F. Mahmood, S.P. Moshokoa, M. Belic, Optik 165, 74 (2018).

[3] A. Biswas, M. Ekici, A. Sonmezoglu, R.T. Alqahtani, Optik 165, 102 (2018).

[4] A. Biswas, H. Rezazadeh, M. Mirzazadeh, M. Eslami, M. Ekici, Q. Zhou, S.P. Moshokoa, M. Belic, Optik 165, 288 (2018).

[5] J.H. Lee, C.K. Lin, O.K. Pashaev, Chaos Solitons Fractals 19, 109 (2004).

[6] A.H. Bhrawy, A.A. Alshaery, E.M. Hilal, D. Milovic, L. Moraru, M. Savescu, A. Biswas, Proc. Rom. Acad. Ser. A 15, 235 (2014).

[7] A. Biswas, D. Milovic, Commun. Nonlinear Sci. Numer. Simulat. 14, 1993 (2009).

[8] A. Biswas, M. Mirzazadeh, M. Eslami, D. Milovic, M. Belic, Frequenz 68, 525 (2014). 
[9] N. Cheemaa, M. Younis, Waves Random Complex Media 26, 30 (2016).

[10] N. Cheemaa, M. Younis, Nonlin. Dynam. 83, 1395 (2016).

[11] G.P. Agarwal, Nonlinear Fiber Optics, Academic, New York 2001.

[12] A. Biswas, K.R. Khan, M.F. Mahmood, M. Belic, Optik 125, 3299 (2014)

[13] A. Biswas, M. Mirzazadeh, M. Savescu, D. Milovic, K.R. Khan, M.F. Mahmood, M. Belic, J. Mod. Opt. 61, 1550 (2014).

[14] A. Biswas, M.Z. Ullah, M. Asma, Q. Zhou, S.P. Moshokoa, M. Belic, Optik 139, 16 (2017).

[15] M. Kaplan, Opt. Quant. Electron. 49, 312 (2017).

[16] M. Kaplan, A. Bekir, M.N. Ozer, Opt. Quant. Electron. 49, 266 (2017).

[17] M. Ekici, M. Mirzazadeh, M. Eslami, Nonlin. Dynam. 84, 669 (2016).

[18] E.V. Krishnan, M. Al Ghabshi, Q. Zhou, K.R. Khan, M.F. Mahmood, Yanan Xu, A. Biswas, J. Optoelectron. Adv. Mater. 17, 511 (2015).

[19] M. Eslami, M. Mirzazadeh, A. Biswas, J. Mod. Opt. 60, 1627 (2013).

[20] N. Raza, A. Javid, Optik 158, 1049 (2018).
[21] M. Mirzazadeh, A.H. Arnous, M.F. Mahmood, E. Zerrad, A. Biswas, Nonlin. Dynam. 81, 277 (2015).

[22] A. Sonmezoglu, M. Yao, M. Ekici, M. Mirzazadeh, Q. Zhou, Nonlin. Dynam. 88, 595 (2017).

[23] H. Triki, A. Biswas, S.P. Moshokoa, M. Belic, Optik 128, 63 (2017)

[24] M. Kaplan, A. Akbulut, A. Bekir, Commun. Theor. Phys. 65, 563 (2016).

[25] J. Manafian, M. Lakestani, Europ. Phys. J. Plus 130, $61(2015)$

[26] J. Manafian, Europ. Phys. J. Plus 130, 255 (2015).

[27] J. Manafian, M. Lakestani, Optik 127, 2040 (2016).

[28] Q. Zhou, M. Ekici, A. Sonmezoglu, J. Manafian, S. Khaleghizadeh, M. Mirzazadeh, Optik 127, 12085 (2016)

[29] H. Bulut, T.A. Sulaiman, H.M. Baskonus, Opt. Quant. Electron. 50, 87 (2018).

[30] H.M. Baskonus, H. Bulut, Waves Random Complex Media 26, 201 (2016).

[31] J. Manafian, J. Jalali, A. Ranjbaran, Opt. Quant. Electron. 49, 1 (2017).

[32] R.F. Zinati, J. Manafian, Eur. Phys. J. Plus 132, 1 (2017). 